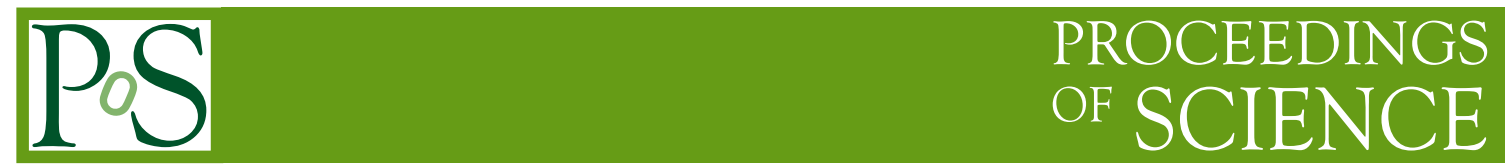

\title{
Electroweak corrections in top-quark physics (1)
}

\section{Johann Kühn ${ }^{a}$, Peter Uwer*b}

${ }^{a}$ Institut für Theoretische Teilchenphysik, Karlsruhe Institute of Technology (KIT)

76128 Karlsruhe, Germany

${ }^{b}$ Institut für Physik, Humboldt-Universität zu Berlin,

12489 Berlin, Germany

E-mail: Johann.Kuehn@KIT.edu, Peter.Uwer@physik.HU-Berlin.de

Electroweak corrections for inclusive top-quark pair production are reviewed and updated results for the weak corrections taking into account the recently measured Higgs mass are presented.

8th International Workshop on Top Quark Physics

14-18 September, 2015

Ischia, Italy

${ }^{*}$ Speaker. 


\section{Introduction}

With the start of the LHC, significant progress has been made concerning top-quark physics. The experimental precision of the inclusive cross section for top-quark pair production is now at the level of a few per cent. At the same time also theoretical predictions within QCD have been significantly improved by the calculation of the next-to-next-to-leading order QCD corrections $[1,2]$. The theoretical uncertainties due to uncalculated higher orders, uncertainties of the parton distribution functions (PDFs), and uncertainties of the QCD coupling constant $\alpha_{s}$ as well as the top-quark mass lead to a total uncertainty of about 3\%. At this level of accuracy electroweak corrections need to be considered as well. From a naive power counting one expects that $\alpha \alpha_{s}$ corrections, with $\alpha$ denoting the electromagnetic coupling, can lead to corrections of the order of $10 \%$, while the pure electroweak corrections of order $\alpha^{2}$ and the mixed corrections of order $\alpha \alpha_{s}^{2}$ may give percentage corrections. A more detailed analysis shows that, because of colour, only very few and kinematically suppressed partonic channels contribute at order $\alpha \alpha_{s}$ (e.g. $\gamma g \rightarrow t \bar{t}$, $b \bar{b} \rightarrow t \bar{t}$ ) leading to a further PDF suppression. In case of the pure electroweak corrections $\left(\alpha^{2}\right)$ the quark charges and the PDF's lead to a further suppression leaving the mixed corrections of order $\alpha \alpha_{s}^{2}$ as the dominant contribution. They can be further 'enhanced' by large Sudakov logarithms. Weak Sudakov logarithms can lead to large negative contributions at large momentum transfer. Inclusive quantities are typically dominated by the threshold region, and are thus less sensitive to Sudakov type corrections. However, differential cross sections at large momentum transfer can be significantly modified. This can affect new physics searches if not taken into account: the possible raise of the cross section due to a (hypothetical) new resonance could be shadowed by the negative weak corrections if not considered in the analysis. Similarly, using spin observables for new physics searches, weak corrections can play an important role since spin observables which are zero in QCD due to parity invariance are not protected to receive weak corrections. At the production threshold of the top-quark pair, the attractive top-Higgs Yukawa interaction leads to an enhancement of the cross section. A detailed study of this effect may provide useful information to constrain the topquark Yukawa coupling. For future experimental investigations it is thus very important to take electroweak corrections into account. Since QED corrections form a gauge invariant contribution, traditionally weak [3-8] and QED [9] corrections were studied separately. In the next section we will briefly discuss the impact of the weak corrections using up to date input for the top-quark mass and the Higgs boson mass. In section 3 we review the status of the QED corrections. It is worth noting that similar to QCD corrections also electroweak corrections have recently witnessed tremendous progress concerning the automated evaluation (see for example Refs. [10-14]).

\section{Impact of weak corrections}

For details on the calculation of the weak corrections to top-quark pair production we refer to Refs. [3-8]. In Ref. [8] the numerical results have been updated using the recently measured value of the Higgs mass. In Fig. 1 (left) the corrections to the inclusive cross section is shown as function of the cms energy. Consistent with the naive expectations the relative corrections are of the order of $-1.8 \%$ at $7 \mathrm{TeV}$ reaching slightly less than $-2.1 \%$ at $30 \mathrm{TeV}$. For comparison, results using a Higgs mass of $1 \mathrm{TeV}$ are shown, illustrating that the Higgs contribution leads to a positive correction to the 

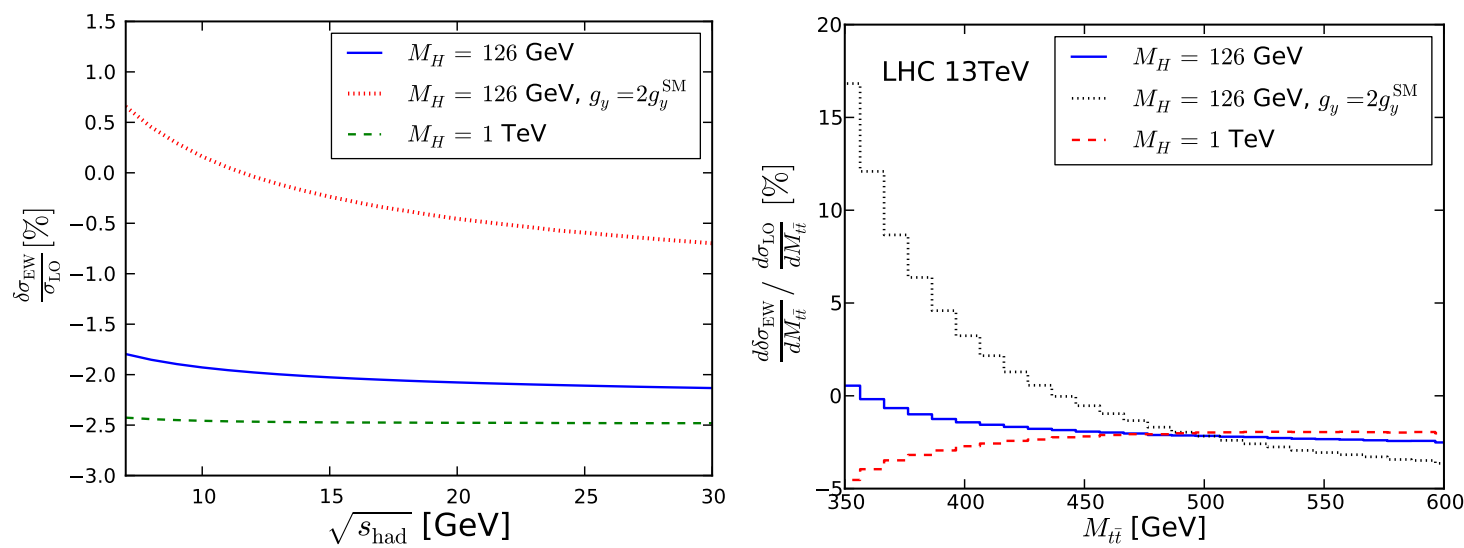

Figure 1: Impact of the weak correction on the inclusive cross section for top-quark pair production as a function of the center of mass energy (left). Threshold effects due to attractive top-Higgs Yukawa interaction (right).

cross section of about $0.5 \%$. Indeed a naive rescaling of the top-quark Yukawa coupling by a factor of 4 leads to a cross section which is about $2 \%$ larger. The right plot in Fig. 1 shows the sensitivity of the threshold region on the top-quark Yukawa interaction. At the threshold, the Higgs corrections lead to a positive correction to the cross section of about 5\%. A two times larger top-quark Yukawa coupling would thus lead to $20 \%$ larger corrections. Experimental studies of the threshold region can thus provide important constraints on the top-quark Yukawa coupling. We also note that the size of the effects is perfectly consistent with the theoretical estimate based on the Sommerfeld enhancement, for details we refer to Ref. [8]. In Fig. 2 the Sudakov suppression at large momentum
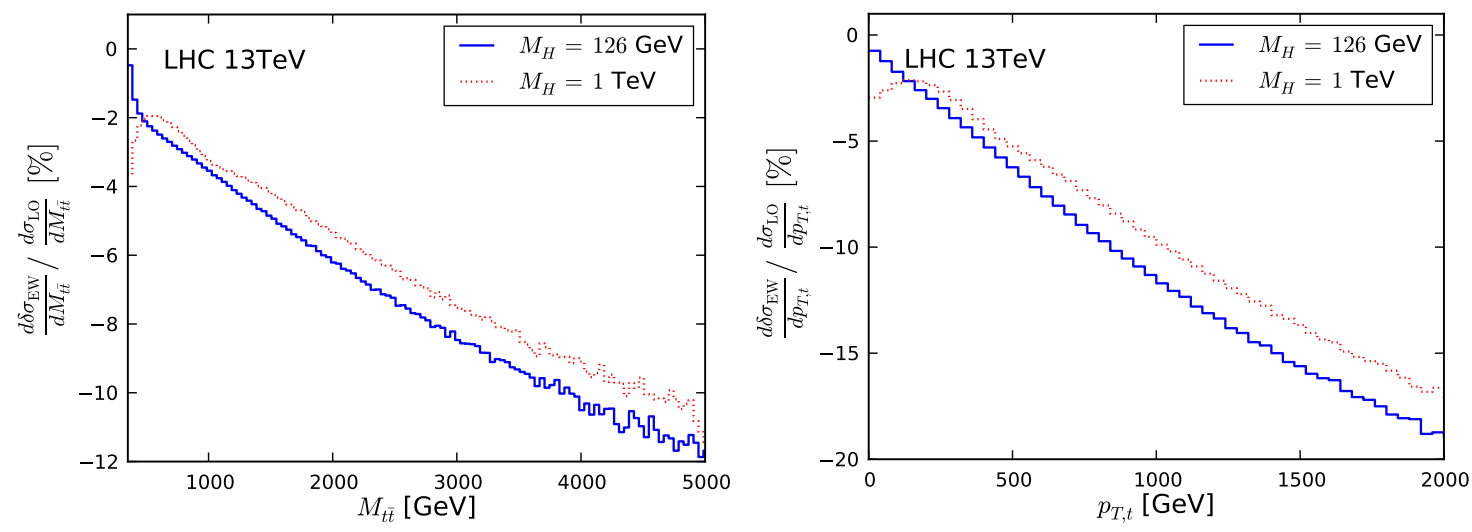

Figure 2: Sudakov suppression at large momentum transfer.

transfer is illustrated. At a transverse momentum of $1 \mathrm{TeV}$ the Sudakov logarithms lead to a relative suppression of about $10 \%$. Since the structure of the Sudakov logarithms is known, one may use this information to produce approximated predictions for the weak corrections. This has been done in Ref. [14] to study weak corrections for top-quark pair production with the program Monte Carlo for FeMto barn processes (MCFM). While the approximation works reasonably well for the $q \bar{q}$ 
partonic channel, the naive approximation fails for the $g g$ channel. Consistent with the findings of Ref. [8] this has been traced back in Ref. [14] to the more complicated angular dependence in the $g g$ channel. As pointed out in Ref. [8] the Sudakov limit in gluon fusion requires the partonic Mandelstam variable $\hat{t}$ or $\hat{u}$ to be large. Indeed it has been shown in Ref. [14] that the Sudakov approximation can be improved by an additional cut on the top-quark rapidity. The origin of the Sudakov logarithms are soft- and collinear configurations which are regulated by the gauge boson masses. For massless gauge bosons the corresponding singularities would require the inclusion of real emission processes to cancel the singularities. In case of massive gauge bosons it is argued that real emission processes lead to a distinguishable finale state. The combination of the real and virtual corrections which could lead to a partial cancellation of the would-be singularities is thus not performed. In Ref. [15] it has been pointed out that at very high energies, the clear separation between states with and without real emission might no longer be possible, thus requiring at least a partial combination of the two. The positive contribution from the real corrections can than partially compensate the negative Sudakov logarithms. With the high energetic events available at the LHC Run II, the analysis of Ref. [15] should be updated using an up-to-date setup. To facilitate the experimental analysis, the Sudakov enhanced corrections have been implemented recently in an updated version of the HATHOR program [16]. In addition, Ref. [8] also describes a prescription to combine the weak corrections with parton shower results.

\section{QED corrections}

The QED corrections to top-quark pair prodcution were first calculated in Ref. [9]. The results for the inclusive cross section as evaluated in Ref. [9] are shown in Tab. $1^{1}$. In Ref. [9] the MRST2004 PDF set has been used and the factorization scale $\mu$ is set to twice the top-quark mass $m_{t}$. While the overall size of the corrections agree well with the naive estimates given in the introduction, the contribution from $g \gamma$ turns out to be quite large, taking into account that the photon PDF should be supressed by an additional factor $\alpha$. Furthermore, a significant cancellation between the quark-anti-quark initiated processes and the $g \gamma, g g$ channels can be observed. In case of the $g \gamma$ channel the price of an additional factor $\alpha$ in the photon PDF seems to be compensated by the large $q g$ luminosity. In Ref. [9] also distributions are investigated. For the transverse momentum distribution, the corrections are positive below $500 \mathrm{GeV}$. Above $500 \mathrm{GeV}$ the corrections are negative and amount to $-6 \%$ at a transverse momentum of $3 \mathrm{TeV}$. In Ref. [9] it is argued that the negative corrections at large transverse momentum are due to logarithmically enhanced final state corrections. Given the progress concerning the PDF extraction it will be important for future analysis to study the impact of the recently published NNPDF and CTEQ14QED PDF sets. In addition, in view of a possible combination of the QED corrections with the QCD results, it would be useful to update the results to the commonly used scale setting $\mu=m_{t}$. First results in this direction have been prepared by Pagani and Zaro using Ref. [13] and are shown in Fig. 3. As can be seen from Fig. 3 the QED corrections lead to a partial compensation of the weak corrections, such that the sum of the two is almost zero, however, at the price of introducing a large PDF uncertainty which is shown as a band in the lowest plot. As PDF set NNPDF2.3QED has been used. Further stud-

\footnotetext{
${ }^{1}$ In Ref. [9] the centre-of-mass energy is not given, most likely $14 \mathrm{TeV}$ has been used.
} 


\begin{tabular}{lcl}
\hline \hline \multicolumn{3}{c}{$\sigma_{\text {tot }}$ without cuts [pb] } \\
Process & Born & Correction \\
\hline$u \bar{u}$ & 34.25 & -1.41 \\
$d \bar{d}$ & 21.61 & -0.228 \\
$s \bar{s}$ & 4.682 & -0.0410 \\
$c \bar{c}$ & 2.075 & -0.0762 \\
$g g$ & 407.8 & 2.08 \\
$g \gamma$ & & 4.45 \\
$p p$ & 470.4 & 4.78 \\
\hline \hline
\end{tabular}

Table 1: QED corrections to inclusive top-quark pair production [9].

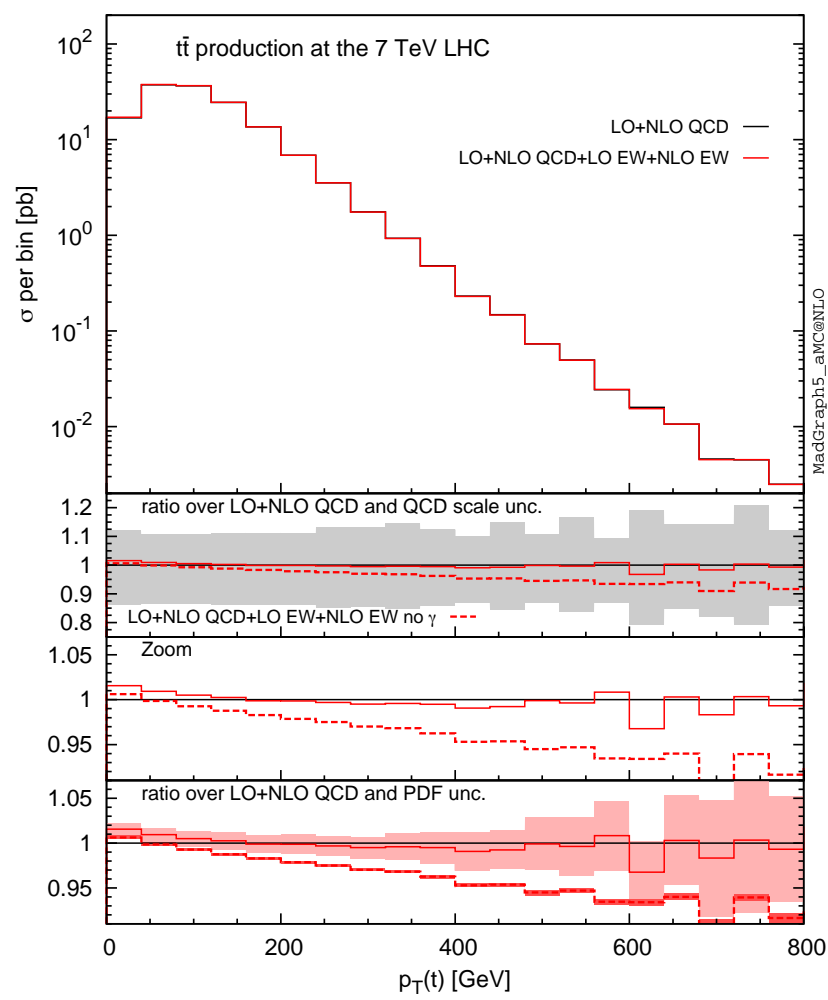

Figure 3: Comparison of QED and weak corrections, prepared by Davide Pagani and Marco Zaro using MadGraph5_aMC@NLO [13].

ies are required to compare with CTEQ14QED and investigate the behavior at significantly larger momentum transfer.

\section{Conclusion}

The experimental precision of cross section measurements for top-quark pair production currently achieved requires the inclusion of electroweak corrections. The weak corrections to the inclu- 
sive cross section amount to about $-2 \%$ which is of the order of the theoretical uncertainty quoted for the NNLO QCD corrections. At threshold the enhancement due to the attractive Yukawa interaction may provide a mean to constrain the top-quark Yukawa coupling. At large momentum transfer the corrections - if not taken into account-may hide a possible raise of the cross section due to new physics resonances at large invariant mass. The QED corrections can also lead to percentage corrections. However, recent studies indicate that the photon PDF's introduce a significant source of uncertainty in the theoretical predictions.

\section{References}

[1] M. Czakon, P. Fiedler, and A. Mitov, Total Top-Quark Pair-Production Cross Section at Hadron Colliders Through $O\left(\alpha_{s}^{4}\right)$, Phys. Rev. Lett. 110 (2013) 252004, [arXiv:1303.6254].

[2] M. Czakon, D. Heymes, and A. Mitov, High-precision differential predictions for top-quark pairs at the LHC, arXiv: 1511.00549.

[3] W. Beenakker, A. Denner, W. Hollik, R. Mertig, T. Sack, and D. Wackeroth, Electroweak one loop contributions to top pair production in hadron colliders, Nucl. Phys. B411 (1994) 343-380.

[4] J. H. Kühn, A. Scharf, and P. Uwer, Electroweak corrections to top-quark pair production in quark-antiquark annihilation, Eur. Phys. J. C45 (2006) 139-150, [hep-ph/ 0508092 ].

[5] W. Bernreuther, M. Fuecker, and Z. G. Si, Mixed QCD and weak corrections to top quark pair production at hadron colliders, Phys. Lett. B633 (2006) 54-60, [hep-ph/ 0508091$].$

[6] J. H. Kühn, A. Scharf, and P. Uwer, Electroweak effects in top-quark pair production at hadron colliders, Eur. Phys. J. C51 (2007) 37-53, [hep-ph/ 0610335$].$

[7] W. Bernreuther, M. Fuecker, and Z.-G. Si, Weak interaction corrections to hadronic top quark pair production, Phys. Rev. D74 (2006) 113005, [hep-ph/ 0610334 ].

[8] J. H. Kühn, A. Scharf, and P. Uwer, Weak Interactions in Top-Quark Pair Production at Hadron Colliders: An Update, Phys. Rev. D91 (2015), no. 1 014020, [arXiv: 1305.5773].

[9] W. Hollik and M. Kollar, NLO QED contributions to top-pair production at hadron collider, Phys. Rev. D77 (2008) 014008, [arXiv: 0708 .1697].

[10] M. Chiesa, N. Greiner, and F. Tramontano, Electroweak corrections for LHC processes, arXiv:1507.08579.

[11] D. Pagani, The electroweak contribution to top quark pair production: cross sections and asymmetries, in 7th Workshop on the CKM Unitarity Triangle (CKM 2012) Cincinnati, Ohio, USA, September 28-October 2, 2012, 2012. arXiv: 1212.0808.

[12] S. Kallweit, J. M. Lindert, P. Maierhöfer, S. Pozzorini, and M. Schönherr, NLO electroweak automation and precise predictions for W+multijet production at the LHC, JHEP 04 (2015) 012, [arXiv:1412.5157].

[13] S. Frixione, V. Hirschi, D. Pagani, H. S. Shao, and M. Zaro, Weak corrections to Higgs hadroproduction in association with a top-quark pair, JHEP 09 (2014) 065, [arXiv: 1407.0823 ].

[14] J. M. Campbell, D. Wackeroth, and J. Zhou, Electroweak Corrections at the LHC with MCFM, in Proceedings, 23rd International Workshop on Deep-Inelastic Scattering and Related Subjects (DIS 2015), 2015. arXiv:1508.06247. 
[15] U. Baur, Weak Boson Emission in Hadron Collider Processes, Phys. Rev. D75 (2007) 013005, [hep-ph/0611241].

[16] M. Aliev, H. Lacker, U. Langenfeld, S. Moch, P. Uwer, and M. Wiedermann, HATHOR: HAdronic Top and Heavy quarks crOss section calculatoR, Comput. Phys. Commun. 182 (2011) 1034-1046, [arXiv:1007.1327]. 